ESAIM: PS

February 2007, Vol. 11, p. 80-88

DOI: $10.1051 / \mathrm{ps}: 2007007$
ESAIM: Probability and Statistics

www.edpsciences.org/ps

\title{
PRICING RULES UNDER ASYMMETRIC INFORMATION
}

\author{
Shigeyoshi Ogawa ${ }^{1}$ And Monique Pontier ${ }^{2}$
}

\begin{abstract}
We consider an extension of the Kyle and Back's model [Back, Rev. Finance Stud. 5 (1992) 387-409, Kyle, Econometrica 35 (1985) 1315-1335], meaning a model for the market with a continuous time risky asset and asymmetrical information. There are three financial agents: the market maker, an insider trader (who knows a random variable $V$ which will be revealed at final time) and a non informed agent. Here we assume that the non informed agent is strategic, namely he/she uses a utility function to optimize his/her strategy. Optimal control theory is applied to obtain a pricing rule and to prove the existence of an equilibrium price when the insider trader and the non informed agent are risk-neutral. We will show that if such an equilibrium exists, then the non informed agent's optimal strategy is to do nothing, in other words to be non strategic.
\end{abstract}

Mathematics Subject Classification. 49N30, 60H10, 93E20.

Invited paper accepted September 2005.

\section{INTRODUCTION}

The purpose of this paper is to extend Kyle and Back's model [3,18]. Firstly in 1985 Kyle [18] defined an equilibrium problem. On a Gaussian financial market in discrete time, there are three agents: a market maker, an insider trader, a non informed agent (noise trader). The market maker has to define a pricing rule in such way that an equilibrium does exist between the traders. Back [3] extended this model to continuous time. Then El Karoui and Cho [5] relaxed the Gaussian hypothesis in Kyle's model using fine tools in stochastic control $[8,10]$. Finally, Cho [6] delivered a new version of Back's model, also relaxing the Gaussian hypothesis. In these four papers, the non informed agent is supposed to be non strategic and so he/she is called "noise trader". As in Cho [6], we like to ask the question: what happens if the non informed agent tries to be strategic instead of being only "a noise trader"?

On such a model, let us mention Guillaume Lasserre's thesis [20] and [21] which extended this problem to multivariate case in continuous time, the agents using a non specified utility function.

Among previous models of insider trading, let us mention [1,2,11-14,19,23]. But these models are quite different: the main tools are enlargement of filtration $[1,2,7,16,17,24]$ and change of probability measure [9].

Finally, Kyle and Back's equilibrium model has to be distinguished from other models such that Arrow-Debreu or Arrow-Radner ones. These equilibrium were studied in an asymmetric information context by Pikovski and Karatzas (no published preprint) and Hillairet in her thesis (cf. also [15]).

Keywords and phrases. Equilibrium, optimal control, asymmetric information.

1 Department of Mathematical Sciences Ritsumeikan University, Kusatsu, Shiga, 525-8577 Japan; ogawa-s@se.ritsumei.ac.jp

2 U.M.R. CNRS C 5583, Laboratoire de statistique et probabilités, Université Paul Sabatier, 118 route de Narbonne, 31062 Toulouse Cedex 04, France; pontier@lsp.ups-tlse.fr

(c) EDP Sciences, SMAI 2007 


\section{THE MODEL}

At time $t \in[0,1]$, the insider trader holds $X_{t}$ units on risky asset, the non informed agent receives a random endowment $I_{t}$ and holds $Z_{t}$. Let $Y_{t}=X_{t}+Z_{t}$, which is observed by the market maker. In order to discover price $P_{t}$ of the risky asset, the following hypotheses are done:

There exists a $C^{1,2}$ function $H$ on $] 0,1\left[\times \mathbb{R}\right.$ such that price $P_{t}$ satisfies:

$$
P_{t}=H\left(t, Y_{t}\right), \forall t, x \mapsto H(t, x) \text { is non decreasing, } \partial_{x} H>0
$$

On the filtered probability space $\left(\Omega,\left(\mathcal{F}_{t}^{B}, t \in[0, T]\right), \mathbb{P}\right)$ associated to $B$, a standard Brownian motion, we get a random variable $V$ independent of $B$ and we suppose that the insider knows $V$ (which could be the price at time 1 , more precisely $\left.V=P_{1+}\right)$ so:

$$
\mathrm{d} X_{t}=\alpha\left(t, Y_{t}, V\right) \mathrm{d} t, X_{o} \in L^{1}(\sigma(V)),
$$

where $\alpha$ is a measurable function such that

$$
\forall x, \alpha(., x, V) \text { is càdlàg and } x \mapsto \alpha(s, x, V) \text { is uniformly Lipschitz, on }[0,1] \times \mathbb{R} \text {. }
$$

We will call such function $f(t, x, v)$ (or $f(t, x)$ resp.) with 3 (or 2 ) variables $(t, x, v)$ (or $(t, x)$ ) regular when it satisfies the same condition $(3)$ in $(t, x)$ for each fixed $v$.

The non informed agent buys $Z_{t}$ and consumes the remainder of her/his endowment denoted as $E_{t}$ :

$$
\mathrm{d} E_{t}=e\left(t, Y_{t}\right) \mathrm{d} t+\sigma \mathrm{d} B_{t}, \text { where } \sigma>0
$$

where $e$ is regular in the above sense $(3)$, and

$$
\mathrm{d} Z_{t}=-\beta\left(t, Y_{t}\right) \mathrm{d} t+\mathrm{d} E_{t}, Z_{o} \in \mathbb{R}
$$

where $\beta$ is regular. Notice that $\beta$ represents the non informed agent's consumption speed. He/she invests his/her endowment minus his/her consumption.

So, we can introduce the following filtration:

$$
\mathcal{F}=\left(\sigma\left\{V,\left(B_{s}, s \leq t\right)\right\}, t \in[0,1]\right),
$$

obviously, the filtration $\left.\left(\mathcal{F}_{t}\right), t \in[0,1]\right)$ is completed and get right continuous $(c f$. [1]): it satisfies the "usual" properties" (see for instance [22]). We can consider that $\mathcal{F}_{t}$ is the insider's information at time $t$.

More generally, $\mathcal{F}^{M}$ denotes the complete right continuous filtration generated by the process $M$. For instance, $\mathcal{F}^{Y}$ is the market maker's information at time $t$, the public information. Under the hypothesis that $\partial_{t} H>0$, the knowledge on $Y$ and $P$ are the same, hence the filtrations $\mathcal{F}^{Y}$ and $\mathcal{F}^{P}$ are identical.

Proposition 2.1. Under hypotheses (1) to (4), the following stochastic differential equation admits a unique strong solution:

$$
\mathrm{d} Y_{t}=\left[\alpha\left(t, Y_{t}, V\right)+e\left(t, Y_{t}\right)-\beta\left(t, Y_{t}\right)\right] \mathrm{d} t+\sigma \mathrm{d} B_{t}, t \in[0, T], Y_{0} \neq 0 .
$$

This solution is an $\mathcal{F}$-Markov process.

Proof. The hypotheses are such that (6) satisfies the existence and uniqueness hypotheses (cf. [22] Th. 6 p. 194 for instance).

This equation is a diffusion equation with an initial $\sigma$-algebra non trivial:

$$
\mathcal{F}_{0}=\cap_{t>0}\left(\sigma(V) \vee \mathcal{F}_{t}^{B}\right)
$$


Corollary 2.2. The filtration generated by the process $Y$ and $V$ is the same as the filtration $\mathcal{F}$ :

$$
\mathcal{F}_{t}=\cap_{s>t}\left(\sigma(V) \vee \mathcal{F}_{s}^{Y}\right) .
$$

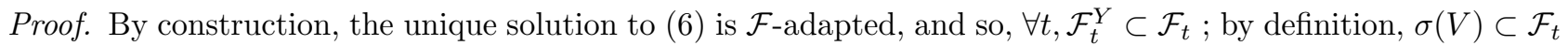
and we get $\cap_{s>t}\left(\mathcal{F}_{s}^{Y} \vee \sigma(V)\right) \subset \cap_{s>t} \mathcal{F}_{s}=\mathcal{F}_{t}$.

Reciprocally, since $\sigma>0$ and

$$
\sigma \mathrm{d} B_{t}=\mathrm{d} Y_{t}-\left[\alpha\left(t, Y_{t}, V\right)+e\left(t, Y_{t}\right)-\beta\left(t, Y_{t}\right)\right] \mathrm{d} t
$$

$\forall t, B_{t}$ is $\mathcal{F}_{t}^{Y} \vee \sigma(V)$-measurable, and we conclude the proof.

Let us remark that the independence between $V$ and $B$ implies that $B$ is also a $(\mathcal{F}, \mathbb{P})$ Brownian motion $(c f$. [7] or [16] for instance). So we get the following:

Proposition 2.3. Conditionally in $V, Y$ is a $\mathcal{F}^{Y}$-Markov process, i.e. $\forall f$ there exists a measurable function $h$ on $[0,1] \times \mathbb{R} \times \mathbb{R}$ such that almost surely:

$$
E\left[\int_{t}^{1} f\left(s, Y_{s}, V\right) \mathrm{d} s / \mathcal{F}_{t}\right]=h\left(t, Y_{t}, V\right) .
$$

Proof. It is a consequence of Proposition 2.1 and Corollary 2.2. We obtain that $Y$ is a $\mathcal{F}$-Markov process, so we get the conclusion.

Remark 2.4. The $\mathcal{F}$-Markov process $Y$ is associated with the infinitesimal generator

$$
\mathcal{A}^{\alpha, \beta}=[\alpha+e-\beta] \partial_{x}+\frac{1}{2} \sigma^{2} \partial_{x x}^{2}
$$

\section{THE PRICING RULE}

The market maker observes the filtration $\mathcal{F}^{Y}$, so that he/she can make price $H\left(t, Y_{t}\right)$.

Definition 3.1. The function $H$, mentioned in the hypothesis (1), is called the pricing rule.

The insider trader has to choose a strategy $\alpha$, and the non informed agent has to choose a strategy $\beta$. The "admissible" strategies $\alpha, \beta$, satisfy Hypotheses (2), (4) and then the stochastic differential equation (6) admits a unique strong solution. So we define:

Definition 3.2. The set of the regular functions $\alpha(t, x, v)$ is denoted by $\mathcal{S}$, whose element is called admissible strategy for the insider trader.

On the other hand, $\mathcal{S}^{\prime}$ the set of the regular functions $\beta(t, x)$, which is the set of admissible strategies for the non insider trader.

Definition 3.3. (1) A strategy $\beta^{*} \in \mathcal{S}^{\prime}$ is optimal if

$$
\beta^{*} \in \operatorname{argmax}\left\{\beta \mapsto E\left[\int_{0}^{1}-P_{s}\left[\left(e\left(s, Y_{s}\right)-\beta\left(s, Y_{s}\right)\right) \mathrm{d} s\right]+\int_{0}^{1} \delta_{s} V(e-\beta)\left(s, Y_{s}\right) \mathrm{d} s\right], \beta \in \mathcal{S}^{\prime}\right\}
$$

where $\delta$ is measurable satisfying $\forall x, \delta(., x)$ is càdlàg and $x \mapsto \delta(s, x)$ is uniformly Lipschitz on [0,1] taking its values in $] 0,1[$.

(2) A strategy $\alpha^{*} \in \mathcal{S}$ is optimal if

$$
\alpha^{*} \in \operatorname{argmax}\left\{\alpha \mapsto E\left[\int_{0}^{1}\left(V-P_{s}\right) \alpha\left(s, Y_{s}, V\right) \mathrm{d} s / \sigma(V)\right], \alpha \in \mathcal{S}\right\} .
$$


Remark that the coefficient $\delta$ is an "impatience coefficient", it means that it delays the agent's profit. Think of [4] where entrepreneur and financiers are differently impatient.

Besides, in [20], the insider's terminal wealth is $W_{0}+\int_{0}^{1} X_{s} \mathrm{~d} P_{s}+\left(V-P_{1}\right) X_{1}$, but using Ito formula this is the same since in our case $\langle X, P\rangle=0$.

The market maker's aim is to discover a pricing rule $H$ satisfying (1) and such that optimal strategies exist in $\mathcal{S}, \mathcal{S}^{\prime}$. Moreover, the price has to be rational ( $P$ is a $\mathcal{F}^{Y}$-martingale):

$$
P_{t}=H\left(t, Y_{t}\right)=E\left[V / \mathcal{F}_{t}^{Y}\right], t \in[0,1[.
$$

Non necessarily $V$ is equal to $P_{1}$ (cf. [3] or [20]), it could be $V=P_{1+}$.

Remark that, as in filtering theory, we can introduce the innovation process which is a $\mathcal{F}^{Y}-$ Brownian motion, i.e.

$$
\mathrm{d} I_{t}=\mathrm{d} B_{t}+\sigma^{-1}(\alpha+e-\beta-\tilde{\alpha})_{t} \mathrm{~d} t \text { where } \tilde{\alpha}_{t}=E_{\mathbb{P}}\left[\alpha\left(t, Y_{t}, V\right) / \mathcal{F}_{t}^{Y}\right]+(e-\beta)\left(t, Y_{t}\right)
$$

in other words $\mathrm{d} Y_{t}=\sigma \mathrm{d} I_{t}+\tilde{\alpha}_{t} \mathrm{~d} t$.

\section{Risk NEUTRAL AGENTS, $u=I d$}

Bellman's principle is to optimize between $t$ and terminal time 1 supposing we know how to optimize between 0 and $t$ (for instance look at [8] p. 95 et sq). Let the value functions:

$$
\begin{aligned}
J^{\alpha}(t)= & \int_{0}^{t}\left(V-H\left(s, Y_{s}\right)\right) \alpha\left(s, Y_{s}, V\right) \mathrm{d} s \\
& +\operatorname{ess} \sup \left\{E\left[\int_{t}^{1}\left(V-P_{s}\right) \gamma\left(s, Y_{s}, V\right) \mathrm{d} s / \mathcal{F}_{t}\right], \gamma \in \mathcal{S}, \gamma \mathbf{1}_{[0, t]}=\alpha \mathbf{1}_{[0, t]}\right\} . \\
J^{\prime \beta}(t)= & E\left[\int_{0}^{t}\left(\delta\left(s, Y_{s}\right) V-P_{s}\right)(e-\beta)\left(s, Y_{s}\right) \mathrm{d} s / \mathcal{F}_{t}^{Y}\right] \\
+ & \left.\operatorname{ess} \sup \left\{E\left[\int_{t}^{1}\left(\delta\left(s, Y_{s}\right) V-P_{s}\right)(e-\gamma)\left(s, Y_{s}\right) \mathrm{d} s\right) / \mathcal{F}_{t}^{Y}\right], \gamma \in \mathcal{S}^{\prime}, \gamma \mathbf{1}_{[0, t]}=\beta \mathbf{1}_{[0, t]}\right\} .
\end{aligned}
$$

Remark that in the first term above $V$ can be get out the integral and replaced by $P_{t}$, its conditional expectation with respect to $\mathcal{F}_{t}^{Y}$.

The following is a consequence of Proposition 2.3.

Proposition 4.1. $\forall \gamma \in \mathcal{S}, \zeta \in \mathcal{S}^{\prime}$, there exist measurable functions $f_{\gamma}, g_{\zeta}$ such that:

$$
\begin{gathered}
E\left[\int_{t}^{1}\left(V-H\left(s, Y_{s}\right)\right) \gamma\left(s, Y_{s}, V\right) \mathrm{d} s / \mathcal{F}_{t}\right]=f_{\gamma}\left(t, Y_{t}, V\right), \\
\left.E\left[\int_{t}^{1}\left(\delta\left(s, Y_{s}\right) V-P_{s}\right)(e-\zeta)\left(s, Y_{s}\right) s \mathrm{~d} s\right) / \mathcal{F}_{t}^{Y}\right]=g_{\zeta}\left(t, Y_{t}\right) .
\end{gathered}
$$

We now denote

$$
W^{\alpha}\left(t, Y_{t}, V\right)=\operatorname{ess} \sup \left\{E\left[\int_{t}^{1}\left(V-H\left(s, Y_{s}\right)\right) \gamma\left(s, Y_{s}, V\right) \mathrm{d} s / \mathcal{F}_{t}\right], \gamma \in \mathcal{S}, \gamma \mathbf{1}_{[0, t]}=\alpha \mathbf{1}_{[0, t]}\right\}
$$

and

$$
\left.U^{\beta}\left(t, Y_{t}\right)=\operatorname{ess} \sup \left\{E\left[\int_{t}^{1}\left(\delta\left(s, Y_{s}\right) V-P_{s}\right)(e-\gamma)\left(s, Y_{s}\right) s \mathrm{~d} s\right) / \mathcal{F}_{t}^{Y}\right], \gamma \in \mathcal{S}, \gamma \mathbf{1}_{[0, t]}=\beta \mathbf{1}_{[0, t]}\right\}
$$


Remark that

and

$$
J^{\alpha}(t)=\int_{0}^{t}\left(V-H\left(s, Y_{s}\right)\right) \alpha \mathrm{d} s+W^{\alpha}\left(t, Y_{t}, V\right)
$$

$$
J^{\prime \beta}(t)=\int_{0}^{t}-H\left(s, Y_{s}\right)(e-\beta)\left(s, Y_{s}\right) \mathrm{d} s+H\left(t, Y_{t}\right) \int_{0}^{t} \delta\left(s, Y_{s}\right)(e-\beta)\left(s, Y_{s}\right) \mathrm{d} s+U^{\beta}\left(t, Y_{t}\right) .
$$

Moreover, we have boundary conditions: $W^{\alpha}(1, x, v)=0, U^{\beta}(1, x)=0, \forall(v, x) \in \mathbb{R}^{2}$.

Let us use Nicole El Karoui's result ([9], Th. 3.10, page 180): $\forall \alpha, \forall \beta, J^{\alpha}$ is a $(\mathcal{F}, \mathbb{P})$ super-martingale and $J^{\prime \beta}$ is a $\left(\mathcal{F}^{Y}, \mathbb{P}\right)$ super-martingale. Moreover, $\alpha^{*} \in \mathcal{S}, \beta^{*} \in \mathcal{S}^{\prime}$ are optimal if and only if $J^{\alpha}$ is a local $(\mathcal{F}, \mathbb{P})$ martingale and $J^{\prime \beta}$ is a local $\left(\mathcal{F}^{Y}, \mathbb{P}\right)$ martingale. So we get a tool to manage the existence of a couple of optimal strategies. With Ito's derivation formula - dropping $\alpha$ and $\beta$ for simplicity and denoting $\delta_{t}(e-\beta)_{t}$ instead of $\delta\left(t, Y_{t}\right)(e-\beta)\left(t, Y_{t}\right)$ - we get:

$$
\begin{aligned}
\mathrm{d} J^{\alpha}(t)= & \left(V-H\left(t, Y_{t}\right)\right) \alpha \mathrm{d} t+\partial_{t} W \mathrm{~d} t+\mathcal{A}^{\alpha, \beta} W \mathrm{~d} t+\sigma \partial_{x} W \mathrm{~d} B_{t} . \\
\mathrm{d} J^{\prime \beta}(s)= & -H\left(t, Y_{t}\right)(e-\beta)_{t} \mathrm{~d} t+H\left(t, Y_{t}\right) \delta_{t}(e-\beta)_{t} \mathrm{~d} t \\
& +\int_{0}^{t} \delta_{s}(e-\beta)_{s} \mathrm{~d} s\left[\partial_{t} H\left(t, Y_{t}\right) \mathrm{d} t+\partial_{x} H\left(t, Y_{t}\right)[\alpha+e-\beta]_{t} \mathrm{~d} t+\frac{1}{2} \partial_{x x}^{2} H \sigma^{2} \mathrm{~d} t\right] \\
& +\partial_{t} U \mathrm{~d} t+\mathcal{A}^{\alpha, \beta} U \mathrm{~d} t+\sigma\left[\partial_{x} U+\int_{0}^{t} \delta_{s}(e-\beta)_{s} \mathrm{~d} s \times \partial_{x} H\left(t, Y_{t}\right)\right] \mathrm{d} B_{t} .
\end{aligned}
$$

We have to write the process $J^{\prime \beta}$ with respect to the $\left(\mathcal{F}^{Y}, \mathbb{P}\right)$-Brownian motion $I$ :

$$
\begin{aligned}
\mathrm{d} J^{\prime \beta}(s)= & -H\left(t, Y_{t}\right)(e-\beta)_{t} \mathrm{~d} t+H\left(t, Y_{t}\right) \delta_{t}(e-\beta)_{t} \mathrm{~d} t \\
& +\int_{0}^{t} \delta_{s}(e-\beta)_{s} \mathrm{~d} s\left[\partial_{t} H+\partial_{x} H \tilde{\alpha}_{t}+\frac{1}{2} \partial_{x x}^{2} H \sigma^{2}\right]\left(t, Y_{t}\right) \mathrm{d} t \\
& +\left[\partial_{t} U+\partial_{x} U \tilde{\alpha}_{t}+\frac{1}{2} \partial_{x x}^{2} U \sigma^{2}\right]\left(t, Y_{t}\right) \mathrm{d} t+\sigma\left[\partial_{x} U+\int_{0}^{t} \delta_{s}(e-\beta)_{s} \mathrm{~d} s \times \partial_{x} H\left(t, Y_{t}\right)\right] \mathrm{d} I_{t} .
\end{aligned}
$$

First of all, the super-martingale property implies the two following inequalities $\forall \alpha, \forall \beta$ :

$$
\begin{aligned}
-H\left(t, Y_{t}\right)(e-\beta)_{t}+ & H\left(t, Y_{t}\right) \delta_{t}(e-\beta)_{t}+\int_{0}^{t} \delta_{s}(e-\beta)_{s} \mathrm{~d} s\left[\partial_{t} H+\partial_{x} H \tilde{\alpha}_{t}+\frac{1}{2} \partial_{x x}^{2} H \sigma^{2}\right]\left(t, Y_{t}\right) \\
+ & {\left[\partial_{t} U+\partial_{x} U \tilde{\alpha}_{t}+\frac{1}{2} \partial_{x x}^{2} U \sigma^{2}\right]\left(t, Y_{t}\right) \leq 0,\left(V-H\left(t, Y_{t}\right)\right) \alpha+\partial_{t} W \mathrm{~d} t+\mathcal{A}^{\alpha, \beta} W \leq 0 . }
\end{aligned}
$$

The first inequality has to be satisfied $\forall \alpha, \forall \beta$, so it has to be $\forall \tilde{\alpha}$. But this expression is linear with respect to $\tilde{\alpha}$. So we get:

$$
\int_{0}^{t} \delta_{s}(e-\beta)_{s} \mathrm{~d} s \times \partial_{x} H\left(t, Y_{t}\right)+\partial_{x} U\left(t, Y_{t}\right)=0
$$

and the optimality of $\beta$ is equivalent to:

$$
\left(\delta_{t}-1\right)(e-\beta)_{t} H\left(t, Y_{t}\right)+\int_{0}^{t} \delta_{s}(e-\beta)_{s} \mathrm{~d} s\left[\partial_{t} H+\frac{1}{2} \partial_{x x}^{2} H \sigma^{2}\right]\left(t, Y_{t}\right)+\left(\partial_{t} U+\frac{1}{2} \sigma^{2} \partial_{x x}^{2} U\right)\left(t, Y_{t}\right)=0 .
$$

Remark that this system implies that $d J^{\prime \beta}$ is identically null, so $J^{\prime \beta}$ is a constant on time. 
Equation (15) implies $\mathrm{d} t \otimes d \mathbb{P}$ almost surely (recall that by hypothesis $\partial_{x} H>0$ ):

$$
\int_{0}^{t} \delta_{s}(e-\beta)_{s} \mathrm{~d} s=-\frac{\partial_{x} U\left(t, Y_{t}\right)}{\partial_{x} H\left(t, Y_{t}\right)}
$$

Differentiate this equation with respect to time:

$$
\begin{aligned}
& \delta_{t}(e-\beta)_{t} \mathrm{~d} t= \\
& \left(-\frac{\partial_{x t}^{2} U}{\partial_{x} H}+\frac{\partial_{x} U \partial_{x t}^{2} H}{\left(\partial_{x} H\right)^{2}}\right)\left(t, Y_{t}\right) \mathrm{d} t-\partial_{x}\left(\frac{\partial_{x} U}{\partial_{x} H}\right)\left(t, Y_{t}\right) \mathrm{d} Y_{t}-\frac{1}{2} \sigma^{2} \partial_{x x}^{2}\left(\frac{\partial_{x} U}{\partial_{x} H}\right)\left(t, Y_{t}\right) \mathrm{d} t .
\end{aligned}
$$

So the local martingale part is null, that is to say, since $Y$ is a Brownian diffusion,

$$
\forall t, \forall x, 0=\partial_{x}\left(\frac{\partial_{x} U}{\partial_{x} H}\right)(t, x)
$$

and consequently $\frac{\partial_{x} U(t, x)}{\partial_{x} H(t, x)}$ does not depend on $x$ so

$$
\forall t, d \mathbb{P} \text { a.s., }(\delta(e-\beta))\left(t, Y_{t}\right)=-\partial_{t}\left(\frac{\partial_{x} U}{\partial_{x} H}\right)\left(t, Y_{t}\right)\left(\text { denoted as }-f^{\prime}(t)\right)
$$

only depends on time, so does the function $\delta(e-\beta)$. Yields from (18) that $\partial_{x} U / \partial_{x} H$ is a function only of time and from (16):

$$
\partial_{x} U(t, x)=[f(t)-f(0)] \partial_{x} H(t, x),
$$

and since $U(1, x)=0 \forall x$ we can conclude that

$$
U(t, x)=[f(t)-f(0)] H(t, x)+g(t) \text { with } f(1)-f(0)=g(1)=0 .
$$

Besides we deduce from $\delta_{t}(e-\beta)_{t}=-f^{\prime}(t)$, that:

$$
(e-\beta)_{t}=-\delta_{t}^{-1} f^{\prime}(t) \text { and } \int_{0}^{t} \delta_{s}(e-\beta)_{s} \mathrm{~d} s=f(0)-f(t) .
$$

We now use Equation (16) and the results in (19), $\forall t, \forall x$ :

$$
\begin{aligned}
0= & -\left(\delta_{t}-1\right) \delta_{t}^{-1} f^{\prime}(t) H(t, x)+(f(0)-f(t))\left(\partial_{t} H+\frac{1}{2} \sigma^{2} \partial_{x x}^{2} H\right)(t, x) \\
& +(f(t)-f(0))\left(\partial_{t} H+\frac{1}{2} \sigma^{2} \partial_{x x}^{2} H\right)(t, x)+f^{\prime}(t) H(t, x)+g^{\prime}(t)
\end{aligned}
$$

and after cancellations, $\forall t, \forall x$ :

$$
\delta^{-1}(t, x) f^{\prime}(t) H(t, x)+g^{\prime}(t)=0 .
$$

Recalling that $\delta^{-1}\left(t, Y_{t}\right) f^{\prime}(t)=-(e-\beta)\left(t, Y_{t}\right)$, this yields the optimal strategy for the non informed agent:

$$
\left(e-\beta^{*}\right)\left(t, Y_{t}\right) H\left(t, Y_{t}\right)=g^{\prime}(t) .
$$

By the way, as in the non insider's case, the super-martingale property:

$$
\forall \alpha,\left(V-H\left(t, Y_{t}\right)\right) \alpha+\partial_{t} W+(\alpha+e-\beta)_{t} \partial_{x} W+\frac{1}{2} \sigma^{2} \partial_{x x}^{2} W \leq 0,
$$


induces a linear expression with respect to $\alpha$, so once again we get:

$$
0=V-H(s, x)+\partial_{x} W(s, x, V)
$$

and there exists optimal $\alpha^{*}$ is equivalent to:

$$
0=\partial_{t} W(s, x, V)+\frac{1}{2} \sigma^{2} \partial_{x x}^{2} W(s, x, V)+(e-\beta)_{t} \partial_{x} W
$$

with boundary condition $W(1, x, V)=0$.

In such a case, we get a necessary and sufficient condition for the existence of an optimal $\alpha^{*}$ is the system:

$$
\begin{aligned}
& \partial_{x} W(s, x, V)=H(s, x)-V, \partial_{x x}^{2} W(s, x, V)=\partial_{x} H(s, x), \\
& \partial_{t} W(s, x, V)=-(e-\beta)_{t} \partial_{x} W(s, x, V)-\frac{1}{2} \sigma^{2} \partial_{x x}^{2} W(s, x, V),
\end{aligned}
$$

thus using the expression (19) for $(e-\beta)$ yields:

$$
\partial_{t} W=-H^{-1}(s, x) g^{\prime}(t)(H(s, x)-V)-\frac{1}{2} \sigma^{2} \partial_{x} H(s, x)=-g^{\prime}(t)+g^{\prime}(t) H^{-1} V-\frac{1}{2} \sigma^{2} \partial_{x} H .
$$

We differentiate this last equation with respect to $x$ and (25) with respect to time $t$, so we get:

$$
\partial_{t} H=\partial_{x t}^{2} W=-g^{\prime}(t) H^{-2} V \partial_{x} H-\frac{1}{2} \sigma^{2} \partial_{x x}^{2} H
$$

and a nonlinear differential equation:

$$
\partial_{t} H=-g^{\prime}(t) H^{-2} V \partial_{x} H-\frac{1}{2} \sigma^{2} \partial_{x x}^{2} H .
$$

But this one is depending on $V$, so necessarily $g^{\prime}$ is identically null and thus, recalling $(20), f^{\prime}=0$ and the optimal strategy is $(e-\beta)\left(t, Y_{t}\right)=0$, that is to say

\section{the better the noise trader has to do is to do nothing.}

So we now came back to Cho's paper [6]. But we have stress that a non informed trader has not to invest to do the best.

Using Ito formula, we get $H\left(t, Y_{t}\right)$ as a semi-martingale, but by definition, $t \mapsto H\left(t, Y_{t}\right)$ is a $\left(\mathcal{F}^{Y}, \mathbb{P}\right)$-martingale so it has to be driven by the innovation process $I$ :

$$
\mathrm{d} H\left(t, Y_{t}\right)=\left[\partial_{t} H+\tilde{\alpha}_{t} \partial_{x} H+\frac{1}{2} \sigma^{2} \partial_{x x}^{2} H\right]\left(t, Y_{t}\right) \mathrm{d} t+\sigma \partial_{x} H\left(t, Y_{t}\right) \mathrm{d} I_{t},
$$

thus $t \mapsto H\left(t, Y_{t}\right)$ is a $\left(\mathcal{F}^{Y}, \mathbb{P}\right)$-martingale is equivalent to a new partial differential equation

$$
\partial_{t} H+\tilde{\alpha}_{t} \partial_{x} H+\frac{1}{2} \sigma^{2} \partial_{x x}^{2} H=0 .
$$

The comparison with the previous one (27) shows that actually if $\alpha$ is optimal, $\tilde{\alpha}$ has to be null, i.e. a result shown by Cho. Remark that in such a case $\mathrm{d} Y_{t}=\sigma \mathrm{d} I_{t}$ and $\sigma^{-1} Y$ is a $\left(\mathcal{F}^{Y}, \mathbb{P}\right)$ Brownian motion. 


\section{Modification}

So far we have discussed the problem in its simplified situation, that is: we supposed the market maker observes the sum $Y_{t}=X_{t}+Z_{t}$ to make price. In other words we developed the discussion by treating the intensity of both traders' activities, insider and non-informed, with equal weight. But there may be an idea saying that such situation may not be close to the reality. Let us consider for example an extreme case where the activity $X_{t}$ of the insider is very small and almost negligeable before the overwhelming activity of majority noise traders. Then we wonder if our result can still hold true for such case.

As a first step toward the amelioration in this point of our model, we try to take into acount the portion between the intensities of these two traders, say $A(\in] 0,1[)$ and we suppose that for the price making the market maker observes the amount,

$$
Y_{t}=A X_{t}+(1-A) Z_{t}
$$

Combining this with the equations $(2),(4)$ we find the following equation for the $Y_{t}$, instead of the equation (6).

$$
\mathrm{d} Y_{t}=\left[A \alpha\left(t, Y_{t}, V\right)+(1-A)\left\{e\left(t, Y_{t}\right)-\beta\left(t, Y_{t}\right)\right\}\right] \mathrm{d} t+(1-A) \sigma \mathrm{d} B_{t}, \quad Y_{0} \neq 0
$$

Now with this model we like to repeat the discussion. But this is quite easy because we need not to do other things but to follow the same discussion only by changing the coefficients $\alpha(t, y, v),(e-\beta)(t, y), \sigma$ to the $A \alpha(t, y, v),(1-A)(e-\beta)(t, y),(1-A) \sigma$ respectively. By consequence we readily find that our result about the optimal policy for noise trader (28) still holds true in this modified model, but the equation (29) for the price function should be modified into the following form;

$$
\partial_{t} H+A \tilde{\alpha}_{t} \partial_{x} H+\frac{1}{2}(1-A)^{2} \sigma^{2} \partial_{x x}^{2} H=0 .
$$

Recall that $\tilde{\alpha}_{t}=E\left[\alpha\left(t, Y_{t}, V\right) / \mathcal{F}_{t}^{Y}\right]$ so actually $A \tilde{\alpha}_{t}$ is to be null. Either $A=0$ or $\tilde{\alpha}_{t}=0$. The first case $A=0$ means that there is no insider traders and it is another problem. The alternative is $\tilde{\alpha}_{t}=0$ and we come back to the previous section and Cho's paper [6].

Finally, what happens if $A$ goes to 1 ? meaning that the percent of insider traders is increasing. Then since $\mathrm{d} Y_{t}=A \tilde{\alpha}_{t} \mathrm{~d} t+(1-A) \sigma \mathrm{d} I_{t}$ and the insider's optimal strategy satisfies $\tilde{\alpha}_{t}=0, Y_{t}$ goes to be a constant when $A$ goes to 1. Recalling that $\mathrm{d} Y_{t}=A \alpha_{t} \mathrm{~d} t+(1-A) \sigma \mathrm{d} B_{t}$ it means that also $\alpha$ should be null. This could mean that the existence of noise traders is indispensable for the trading to be done in the market. On the other hand, if $A$ goes to 1, the consequence in Equation (31) is that $\partial_{t} H$ goes to 0 , the price $H$ becomes a constant, and these two facts (constant price and no trading) are consistent.

\section{REFERENCES}

[1] J. Amendinger, Martingale representation theorems for initially enlarged filtrations. Stoch. Proc. Appl. 89 (2000) $101-116$.

[2] J. Amendinger, P. Imkeller and M. Schweizer, Additional logarithmic utility of an insider. Stoch. Proc. Appl. 75 (1998) $263-286$.

[3] K. Back, Insider trading in continuous time. Rev. Financial Stud. 5 (1992) 387-409.

[4] B. Biais, T. Mariotti, G. Plantin and J.C. Rochet, Dynamic security design. Rev. Economic Stud. to appear.

[5] K.H. Cho and N. EL Karoui, Insider trading and nonlinear equilibria:uniqueness: single auction case. Annales d'économie et de statistique 60 (2000) 21-41.

[6] K.H. Cho, Continuous auctions and insider trading: uniqueness and risk aversion. Finance and Stochastics 7 (2003) 47-71.

[7] M. Chaleyat-Maurel and T. Jeulin, Grossissement gaussien de la filtration brownienne, in Séminaire de Calcul Stochastique 1982-83, Paris, Lect. Notes Math. 1118 (1985) 59-109.

[8] N. El Karoui, Les aspects probabilistes du contrôle stochastique, in Ecole d'été de Saint Flour 1979, Lect. Notes Math. 872 (1981) $73-238$.

[9] H. Föllmer and P. Imkeller, Anticipation cancelled by a Girsanov transformation: a paradox on Wiener space. Ann. Inst. Henri Poincaré 29 (1993) 569-586.

[10] W.H. Fleming and R.W. Rishel, Deterministic and Stochastic Optimal Control. Springer, Berlin (1975).

[11] A. Grorud and M. Pontier, Comment détecter le délit d'initié ? CRAS, Sér. 1324 (1997) 1137-1142.

[12] A. Grorud and M. Pontier, Insider trading in a continuous time market model. IJTAF. 1 (1998) 331-347. 
[13] A. Grorud and M. Pontier, Probabilité neutre au risque et asymétrie d'information. CRAS, Sér. 1329 (1999) $1009-1014$.

[14] A. Grorud and M. Pontier, Asymmetrical information and incomplete markets. IJTAF. 4 (2001) 285-302.

[15] C. Hillairet, Existence of an equilibrium with discontinuous prices, asymmetric information and non trivial initial $\sigma$-fields. Math. Finance 15 (2005) 99-117.

[16] J. Jacod, Grossissement initial, Hypothèse H’ et Théorème de Girsanov, in Séminaire de Calcul Stochastique 1982-83, Paris, Lect. Notes Math. 1118 (1985) 15-35.

[17] T. Jeulin, Semi-martingales et grossissement de filtration. Springer-Verlag (1980).

[18] A.S. Kyle, Continuous auctions and insider trading. Econometrica 53 (1985) 1315-1335.

[19] I. Karatzas and I. Pikovsky, Anticipative portfolio optimization. Adv. Appl. Probab. 28 (1996) 1095-1122.

[20] G. Lasserre, Quelques modèles d'équilibre avec asymétrie d'information. Thèse soutenue à l'université de Paris VII, le 16 décembre 2003.

[21] G. Lasserre, Asymmetric information and imperfect competition in a continuous time multivariate security model, Finance and Stochastics 8 (2004) 285-309.

[22] P. Protter, Stochastic Integration and Differential Equations. Springer-Verlag (1990).

[23] M. Schweizer, On the minimal martingale measure and the Föllmer-Schweizer decomposition. Stochastic Anal. Appl. 13 (1995) 573-599.

[24] M. Yor, Grossissement de filtrations et absolue continuité de noyaux, in Séminaire de Calcul Stochastique 1982-83, Paris, Lect Notes Math. 1118 (1985) 6-14. 